\title{
A Beamformer-Particle Filter Framework for Localization of Correlated EEG Sources
}

\author{
Petia Georgieva, Senior Member, IEEE, Nidhal Bouaynaya, Member, IEEE, Filipe Silva, \\ Lyudmila Mihaylova, Senior Member, IEEE, and Lakhmi C. Jain
}

\begin{abstract}
Electroencephalography (EEG)-based brain computer interface (BCI) is the most studied noninvasive interface to build a direct communication pathway between the brain and an external device. However, correlated noises in EEG measurements still constitute a significant challenge. Alternatively, building BCIs based on filtered brain activity source signals instead of using their surface projections, obtained from the noisy EEG signals, is a promising and not well-explored direction. In this context, finding the locations and waveforms of inner brain sources represents a crucial task for advancing source-based noninvasive BCI technologies. In this paper, we propose a novel multicore beamformer particle filter (multicore BPF) to estimate the EEG brain source spatial locations and their corresponding waveforms. In contrast to conventional (single-core) beamforming spatial filters, the developed multicore BPF considers explicitly temporal correlation among the estimated brain sources by suppressing activation from regions with interfering coherent sources. The hybrid multicore BPF brings together the advantages of both deterministic and Bayesian inverse problem algorithms in order to improve the estimation accuracy. It solves the brain activity localization problem without prior information about approximate areas of source locations. Moreover, the multicore BPF reduces the dimensionality of the problem to half compared with the PF solution, thus alleviating the curse of dimensionality problem. The results, based on generated and real EEG data, show that the proposed framework recovers correctly the dominant sources of brain activity.
\end{abstract}

Index Terms-Bayesian estimation, EEG inverse problem, multicore beamformer, particle filtering, spatial-temporal brain source localization.

Manuscript received October 6, 2014; revised February 6, 2015; accepted March 7, 2015. Date of publication; date of current version. This work was supported by the Operational Program Competitiveness Factors-Compete and by National Funds through FCT - Foundation for Science and Technology under the Project FCOMP-01-0124-FEDER-022682 (FCT reference PEst-C/EEI/UI0127/2011) and the U.K. Engineering and Physical Sciences Research Council under the Bayesian Tracking and Reasoning over Time Grant EP/K021516/1 and the USA National Institute Of General Medical Sciences under Award R01GM096191.

P. Georgieva and F. Silva are with the Institute of Electronics Engineering and Telematics of Aveiro (IEETA), Department of Electronics, Telecommunications and Informatics, University of Aveiro, 3810-193 Aveiro, Portugal (e-mail: petia@ua.pt; fmsilva@ua.pt).

N. Bouaynaya is with the Department of Electrical and Computer Engineering, Rowan University, Glassboro, NJ 08028 USA (e-mail: bouaynaya@ rowan.edu)

L. Mihaylova is with the Department of Automatic Control and Systems Engineering, University of Sheffield, Sheffield S10 2TN, U.K. (e-mail: 1.s.mihaylova@sheffield.ac.uk).

L. C. Jain is with the University of South Australia, Adelaide, SA 5001, Australia (e-mail: lakhmi.jain@unisa.edu.au).

Color versions of one or more of the figures in this paper are available online at http://ieeexplore.ieee.org.

Digital Object Identifier 10.1109/JBHI.2015.2413752

\section{INTRODUCTION}

$\mathbf{E}$ LECTROENCEPHALOGRAPHY (EEG) is a widely used technology for brain study because it is noninvasive, relatively cheap, portable and with an excellent temporal resolution. These salient features hold the promise of EEG-based brain computer interface (BCI) technologies [1] capable of building alternative communication channels between humans and the external world. The spatial-temporal reconstruction of the underlying brain neural generators based on the EEG recording has emerged as an active area of research over the last decade.

Several source reconstruction approaches, each employing a different set of assumptions, have been proposed to overcome the ill-posed inverse problem. They can be divided in two main classes, [2]: 1) imaging models (also known as current density reconstruction models), which explain the data with a dense set of current dipoles distributed at fixed locations; and 2) equivalent current dipole models (also known as point source or parametric models), which assume a small number of focal sources at locations to be estimated from the data.

While the imaging techniques provide a detailed map of the neuronal activity, the parametric models represent a direct mapping from scalp topology to a small number of parameters. Dipole solutions provide more intuitive interpretations that explain the sensor data. Furthermore, it is easy to report statistics of dipole parameters over different subjects. Summarizing distributed brain activity with a small number of active dipoles simplifies the analysis of connectivity among those sources. Additionally, building BCIs based on the neuronal sources instead of the EEG sensor data is gaining more interest [3]-[6]. In particular, source-based BCI seems an appealing alternative to well-known invasive solutions through implant placement (intracortical electrodes) by neurosurgery.

Popular deterministic parametric solutions include the multiple signal classification (MUSIC) algorithm and its modified versions [7], the methods for inverse problems [8], the construction of spatial filters by data-independent [9] or data-driven methods [10] and blind source separation techniques [11], [12]. However, these approaches are based on the assumption that the brain source locations are known a priori or perform a search of the overall head volume to find their positions. Given the spatial source locations, they estimate the amplitudes and directions of the source waveforms.

Recently, statistical methods have gained popularity. Galka et al. [2] consider the inverse problem as a dynamical one and apply Kalman filtering to a linear distributed EEG source model. In [13], a variational Bayesian approach is developed, which allows for specification of priors on all the model parameters. 
Following a similar approach as [2], Sorrentino et al. [14], [15] propose a dynamical Bayesian framework to estimate the locations of magnetoencephalografic sources.

In the statistical state-space model framework, the EEG source localization problem is formulated as the estimation of the posterior probability density function (pdf) of the state based on the available observations. For the linear and Gaussian estimation problem, the Kalman filter propagates and updates the mean and covariance of the distribution. For nonlinear problems and non-Gaussian noise, there is no general analytical solution to the posterior density estimation problem. Therefore, a numerical approach is needed to evaluate the posterior pdf of the state vector. The particle filter [16], [17] has emerged, within the object tracking community, as one of the most successful methods for state estimation in highly nonlinear or non-Gaussian statespace models. The main idea is to represent the posterior pdf as a set of random samples, called particles. When propagated and weighted properly, these samples tend to the exact pdf as the number of samples becomes very large [17]. The recent surveys [17]-[20] show successful applications of particle filtering to different areas.

The computational complexity is a major challenge in the reviewed techniques for brain source analysis. It is related with the number of estimated dipoles, the dimension of the dipole grid over which the active dipoles are searched and the dipole temporal correlation. Deterministic solutions like the MUSIC algorithm and its variations look for a tradeoff between the dense dipole grid space and correct dipole estimation of an unlimited number of uncorrelated dipoles, or large grid spacing (for fast computation), but sources may be missed or incorrectly estimated if their true location is too far apart from a grid point. Spatial filters like beamforming (BF) have the advantage of providing closed-form linear solution of the inverse problem [21], [22]. However, they are rather sensitive to correlated dipoles and require knowledge of the dipole positions. Statistical approaches, like particle filtering, are more suitable to the brain source analysis but they have to deal with the problem of the high state vector dimension that usually deteriorates the estimation accuracy. For example, in a full particle filter framework, one estimated dipole corresponds to six estimated parameters (three space location coordinates and three directions of dipole moments propagation). This problem is addressed in [23] by an algorithm that integrates multiple particle filters to estimate individual dipoles. However, such a framework does not provide tools for analyzing a potential dipole correlation and connectivity, which is a central issue in neuroscience. Moreover, applying a full particle filter, designed for solving nonlinear problems, for estimation of dipole moments, a linear function of the EEG signal, is an unjustified complication of the inverse problem.

In order to overcome the limitation of the conventional (single-core) BF [10], [24] to reconstruct only uncorrelated sources, Brookes et al. [25] and Diwakar et al. [26] proposed a dual-core BF to consider two simultaneously activated sources into a single spatial filter. Dalal et al. [27] and Popescu et al. [28] extended the methodology of Diwakar, by adding multiple nullconstraints in the potentially correlated source locations (multicore beamformer). A combined solution of the brain dipole recovery is proposed in [29], referred to as a beamforming particle filter (BPF), where a single-core BF is used to estimate the source waveforms and a particle filter (PF) to estimate the source spatial locations. The algorithm was illustrated for one dipole.

The key contributions of this study are the following: 1) We propose a hybrid approach (multicore BF and PF) that, in contrast to previous solutions, does not assume knowledge of the spatial locations of the brain sources in order to estimate the waveforms. The spatial dipole coordinates are estimated using the PF, whereas the waveforms are estimated using the beamformer; 2) The multicore BF reconstructs the moments of each identified dominant source considering null constraints with respect to the others. Since the number of the identified sources (by PF) is smaller than the suppressed single correlated interferers or nulling entire brain volumes as in previous works [27], [28], the computational complexity of the proposed combined solution is significantly lower; 3) Satisfactory reconstruction accuracy was obtained for very low EEG signal to noise ratios (less than $8 \mathrm{~dB}$ ) which is an additional advantage of the hybrid approach.

This paper is organized as follows: In Section II, the PF framework is outlined. Section III presents the EEG state-space model in order to apply the PF, based on physiological specifications. The beamformer for correlated sources is introduced in Section IV. The joint multicore beamformer and particle filter (multicore BPF), for recursive estimation of the source locations and waveforms is presented in Section V. In Section VI, the multicore BPF is applied to simulated and real EEG data and compared with alternative solutions. Section VII summarizes the results.

\section{PARTICLE FILTER}

The active zones in the brain can be described in general with a nonlinear state space model defined by the state and measurement equations

$$
\begin{aligned}
\boldsymbol{x}_{k+1} & =\boldsymbol{f}_{k}\left(\boldsymbol{x}_{k}, \boldsymbol{w}_{k}\right) \\
\boldsymbol{z}_{k} & =\boldsymbol{h}_{k}\left(\boldsymbol{x}_{k}, \boldsymbol{v}_{k}\right)
\end{aligned}
$$

where $f_{k}$ is the system transition function and $\boldsymbol{w}_{k}$ is a zeromean, white noise sequence of known pdf, independent of past and current states and $k$ is the discrete time index. Measurements $\boldsymbol{z}_{k}, k=1,2, \ldots$, are available at discrete time steps $k$, relating to the unknown state vector $\boldsymbol{x}_{k}$ via the observation equation in (2), where $h_{k}$ is the measurement function and $\boldsymbol{v}_{k}$ is a zeromean, white noise sequence of known pdf, independent of past and present states and the system noise.

Within the Bayesian framework, all relevant information about the state vector, given observations $\boldsymbol{Z}_{1: k}=\left\{\boldsymbol{z}_{1}, \ldots, \boldsymbol{z}_{k}\right\}$ up to and including time $k$, can be obtained from the posterior distribution of the state $p\left(\boldsymbol{x}_{k} \mid \boldsymbol{Z}_{1: k}\right)$. This distribution can be obtained recursively in two steps: prediction and update. Suppose that the posterior distribution at the previous time index $k-1$, $p\left(\boldsymbol{x}_{k-1} \mid \boldsymbol{Z}_{1: k-1}\right)$, is available. Then, using the system transition model, we can obtain the prior pdf of the state at time $k$ as 
follows:

$$
p\left(\boldsymbol{x}_{k} \mid \boldsymbol{Z}_{1: k-1}\right)=\int p\left(\boldsymbol{x}_{k} \mid \boldsymbol{x}_{k-1}\right) p\left(\boldsymbol{x}_{k-1} \mid \boldsymbol{Z}_{1: k-1}\right) d \boldsymbol{x}_{k-1}
$$

When a measurement, at time step $k$, is available, the prior is updated via the Bayes rule [30], [31]

$$
p\left(\boldsymbol{x}_{k} \mid \boldsymbol{Z}_{1: k}\right)=\frac{p\left(\boldsymbol{z}_{k} \mid \boldsymbol{x}_{k}\right) p\left(\boldsymbol{x}_{k} \mid \boldsymbol{Z}_{1: k-1}\right)}{p\left(\boldsymbol{z}_{k} \mid \boldsymbol{Z}_{1: k-1}\right)}
$$

where the denominator is a normalizing factor and the conditional pdf of $\boldsymbol{z}_{k}$ given $\boldsymbol{x}_{k}$ is defined by the measurement model in (2).

The recursive equations in (3) and (4) constitute the solution to the Bayesian estimation problem. If the functions $f_{k}$ and $h_{k}$ are linear and the noises $\boldsymbol{w}_{k}$ and $\boldsymbol{v}_{k}$ are Gaussian with known variances, then an analytical solution to the Bayesian recursive estimation problem is given by the well-known Kalman filter [32]. In the EEG source localization problem, however, the measurement function $h_{k}$ is nonlinear, i.e., the EEG measurements $\boldsymbol{z}_{k}$ are nonlinear functions of the source locations $\boldsymbol{x}_{k}$ [33]. The measurement model will be presented in Section III-A.

In order to deal with the nonlinear models and/or nonGaussian noises, two main approaches have been adopted: parametric and nonparametric. The parametric techniques are based on extensions of the Kalman filter by linearizing nonlinear functions around the predicted values [34]. Other Kalman filter variants such as the extended Kalman filter and unscented Kalman filter [35] exist but they can only deal with unimodal distributions. Because of their first-order approximations and unimodal Gaussian assumptions, such extensions find difficulties in dealing with multimodal distributions. Nonparametric methods such as Monte Carlo methods [16], [30], [35] use a set of random samples, called particles, to represent the posterior pdf. The posterior is then approximated by a set of weighted particles (hence the name particle filter) as [16]

$$
p\left(\boldsymbol{x}_{k} \mid \boldsymbol{Z}_{1: k}\right) \approx \sum_{i=1}^{N} \pi_{k}^{(l)} \delta\left(\boldsymbol{x}_{k}-\boldsymbol{x}_{k}^{(l)}\right)
$$

where $N$ is the total number of particles, $\pi_{k}^{(l)}=\frac{w_{k}^{(l)}}{\sum_{l=1}^{N} w_{k}^{(l)}}$ is the normalized weight for particle $l$ at time $k$ and $\delta($.$) is the Dirac$ delta function.

Different methods [19], [20], [35] have been proposed to update the current weights $w_{k}^{(l)}$ based on previous weights $w_{k-1}^{(l)}$ and measurement $\boldsymbol{z}_{k}$. Sampling-importance-resampling is the most popular method where the importance weight of a particle is given by [16], [30], [35]

$$
w_{k}^{(l)}=w_{k-1}^{(l)} \frac{p\left(\boldsymbol{z}_{k} \mid \boldsymbol{x}_{k}^{(l)}\right) p\left(\boldsymbol{x}_{k}^{(l)} \mid \boldsymbol{x}_{k-1}^{(l)}\right)}{q\left(\boldsymbol{x}_{k}^{(l)} \mid \boldsymbol{x}_{k-1}^{(l)}, \boldsymbol{Z}_{1: k}\right)}
$$

where $q\left(\boldsymbol{x}_{k}^{(l)} \mid \boldsymbol{x}_{k-1}^{(l)}, \boldsymbol{Z}_{1: k}\right)$ denotes the importance function from which samples are drawn. The most popular choice [16] for the prior importance function is $q\left(\boldsymbol{x}_{k}^{(l)} \mid \boldsymbol{x}_{k-1}^{(l)}, \boldsymbol{Z}_{1: k}\right)=$ $p\left(\boldsymbol{x}_{k}^{(l)} \mid \boldsymbol{x}_{k-1}^{(l)}\right)$ and it implies that (6) reduces to

$$
w_{k}^{(l)}=w_{k-1}^{(l)} p\left(\boldsymbol{z}_{k} \mid \boldsymbol{x}_{k}^{(l)}\right) .
$$

Given a discrete approximation to the posterior distribution, one can then proceed to a filtered point estimate such as the mean of the state at time $k$

$$
\hat{\boldsymbol{x}}_{k}=\sum_{l=1}^{N} \pi_{k}^{(l)} \boldsymbol{x}_{k}^{(l)} .
$$

The main advantage of the particle filter is that no restrictions are placed on the modeling functions $f_{k}$ and $h_{k}$, or on the distribution of the system and measurement noise. Moreover, the algorithm is quite simple and very easy to implement. However, this increases the computational cost. Notably, it can be implemented on massively parallel computers, raising the possibility of real time operation with very large sample sets.

\section{EEG STATE-SPACE MODEL}

In order to apply the particle filtering framework, we need to define the state-space model of the EEG source localization problem based on physiological constraints. The state vector $\boldsymbol{x}_{k}$, at time $k$, represents the coordinates of the brain sources, or dipoles, within the 3-D head geometry. For example, for two dipoles, the state vector comprises $\left(x_{i k}, y_{i k}, z_{i k}\right)$, the 3 -D coordinates of the $i$ th dipole in the chosen head geometry at time $k, \boldsymbol{x}_{k}=\left[x_{1 k}, y_{1 k}, z_{1 k}, x_{2 k}, y_{2 k}, z_{2 k}\right]^{t}$ and $t$ is the transpose operator. The observation vector $\boldsymbol{z}_{k}$ represents the EEG measurements collected from all sensors at time $k$. The goal is to estimate the brain source locations given the multichannel EEG signal.

\section{A. EEG Measurement Model}

The main source of EEG potentials, measured at the scalp, derive from simultaneous postsynaptic current flows of many neighboring neurons with similar orientations. In particular, these clusters of similar oriented neurons are mainly found in the cortical areas of the brain associated with the pyramidal cells. The total electric current in an activated region is often modeled by a mathematical current dipole with an adequate dipole moment. Additionally, many of those current dipoles representing microscopic current flows with the same orientation can be replaced by an equivalent current dipole [1]. Assuming that the electrical activity of the brain is originated from $M$ dipolar sources, the measured multichannel EEG signal $\boldsymbol{z}_{k}$ from $n_{z}$ sensors at time $k$ can be expressed by

$$
\boldsymbol{z}_{k}=\sum_{m=1}^{M} \boldsymbol{L}_{m}\left(\boldsymbol{x}_{k}(m)\right) \boldsymbol{s}_{k}(m)+\boldsymbol{v}_{k}
$$

where $\boldsymbol{x}_{k}(m)$ is a $3 M \times 1$-dimensional state vector, that represents the spatial source location at time $k, \boldsymbol{L}_{m}\left(\boldsymbol{x}_{k}(m)\right)$ is the $n_{z} \times 3$ leadfield matrix, also called forward matrix, for the $m$ th dipole; $\boldsymbol{s}_{k}(m)$ is a $3 \times 1$-dimensional moment of the $m$ th dipole at time $k$, and $\boldsymbol{v}_{k}$ is a white Gaussian model noise with covariance $\boldsymbol{C}_{v}$. The components of the leadfield matrix $\boldsymbol{L}_{m}$ are nonlinear functions of the dipole localization, electrodes' positions and head geometry [33]. Note also, from (9), that the EEG measurements $\boldsymbol{z}_{k}$ are linear with respect to the dipole moments $\boldsymbol{s}_{k}$ and nonlinear with respect to their spatial locations $\boldsymbol{x}_{k}$. Though 
we are assuming that the number of dipoles $M$ is known, it can be estimated by analysing the structure of the covariance matrix of the observations and using information-theoretic criteria, as presented in [36].

Equation (9), which takes into account $M$ dipoles, can be written in the following concise form:

$$
\boldsymbol{z}_{k}=\boldsymbol{L}\left(\boldsymbol{x}_{k}\right) \boldsymbol{s}_{k}+\boldsymbol{v}_{k}
$$

where $\boldsymbol{x}_{k}=\left[\boldsymbol{x}_{k}(1), \ldots, \boldsymbol{x}_{k}(M)\right]^{t}$ is a $3 M \times 1$ vector, representing the 3-D location coordinates of the $M$ dipoles at time $k$, $\boldsymbol{L}\left(\boldsymbol{x}_{k}\right)=\left[\boldsymbol{L}_{1}\left(\boldsymbol{x}_{k}(1), \ldots, \boldsymbol{L}_{M}\left(\boldsymbol{x}_{k}(M)\right]\right.\right.$ is a $n_{z} \times 3 M$ lead field matrix of the $M$ dipoles at time $k$, and $\boldsymbol{s}_{k}=\left[\boldsymbol{s}_{k}(1), \ldots, \boldsymbol{s}_{k}(M)\right]$ is the $3 M \times 1$ vector of brain source signals in the three directions for the $M$ dipoles. From (10), we can compute the likelihood of each measurement as

$$
\begin{aligned}
& \mathcal{L}\left(\boldsymbol{z}_{k} \mid\left(\boldsymbol{x}_{k}, \boldsymbol{s}_{k}\right)\right) \propto \\
& \exp \left[-\frac{\left(\boldsymbol{z}_{k}-\boldsymbol{L}\left(\boldsymbol{x}_{k}\right) \boldsymbol{s}_{k}\right)^{t} \boldsymbol{C}_{v}^{-1}\left(\boldsymbol{z}_{k}-\boldsymbol{L}\left(\boldsymbol{x}_{k}\right) \boldsymbol{s}_{k}\right)}{2}\right]
\end{aligned}
$$

where $\propto$ denotes "proportional to."

\section{B. EEG State Transition Model}

We assume no a priori knowledge of the source locations. This is in contrast to other studies where a prior information may be available from other brain imaging modalities like magneto resonance images (MRI) or functional MRI. We therefore, assume the state transition to be a random walk (first-order Markov chain) in the source localization space

$$
\boldsymbol{x}_{k}=\boldsymbol{x}_{k-1}+\boldsymbol{w}_{k}
$$

where $\boldsymbol{w}_{k}$ is a zero-mean, Gaussian white noise sequence with covariance $\sigma_{w}^{2} I$. The process $\boldsymbol{w}_{k}$ is assumed to be independent of past and current states. The Gaussian nature of the system and measurement noise is justified by the central limit theorem, because of the numerous sources of noise introduced in EEG measurements: 1) environmental noise, which comes from the power line and bad electrode contacts; 2) physiological noise, which arises from artifacts like the heart rate or eye blinks; and 3) background noise, which is the result of the constant brain activity.

The state-space model of the dipole source localization problem is then given by

$$
\begin{cases}\boldsymbol{x}_{k}=\boldsymbol{x}_{k-1}+\boldsymbol{w}_{k}, & \text { state transition model } \\ \boldsymbol{z}_{k}=\boldsymbol{L}\left(\boldsymbol{x}_{k}\right) \boldsymbol{s}_{k}+\boldsymbol{v}_{k}, & \text { observation model. }\end{cases}
$$

In the above model, the source waveforms $s_{k}$ are not known and they are estimated by the beamforming filter.

\section{Multicore BEAMFORMing FOR CORRELATED SOURCE LOCALIZATION}

The beamforming, originated in radar and sonar field, is a well-known spatial filter for EEG source estimation [24], [37][43]. The BF estimates the source moments $\boldsymbol{s}_{k}$ by applying the following linear operator:

$$
\boldsymbol{s}_{k}=\boldsymbol{W}^{t} \boldsymbol{z}_{k}
$$

where $\boldsymbol{W}^{t}$ is an $n_{z} \times 3 M$ weighting matrix. The ideal filter transmits the signals from the location of interest with a unit gain, while nulling signals from elsewhere (i.e., insensitive to the activity from other brain regions). Among a number of criteria for choosing the optimal matrix $W$, the eigenspaceprojected linearly constrained minimum variance (LCMV) BF gained much interest [39], [44]. The LCMV formulation allocates spatial nulls so as to minimize the contribution to the filter output from sources at locations other than the estimated source [28]. Under the assumption that source moments associated with different sources are temporally uncorrelated, the solution to this minimization problem is given by [10]

$$
\begin{aligned}
& \boldsymbol{W}^{*}=\underset{\boldsymbol{W}}{\operatorname{argmin}} \operatorname{Tr}\left[\boldsymbol{W}^{t} \boldsymbol{C}_{v} \boldsymbol{W}\right] \\
& \text { subject to } \boldsymbol{W}^{t} \boldsymbol{L}\left(\boldsymbol{x}_{k}\right)=\boldsymbol{I} .
\end{aligned}
$$

The optimal solution is derived by constrained minimization using Lagrange multipliers

$$
\boldsymbol{W}^{*}=\boldsymbol{C}_{v}^{-1} \boldsymbol{L}\left(\boldsymbol{x}_{k}\right)\left[\boldsymbol{L}\left(\boldsymbol{x}_{k}\right)^{t} \boldsymbol{C}_{v}^{-1} \boldsymbol{L}\left(\boldsymbol{x}_{k}\right)\right]^{-1} .
$$

The conventional (single-core) LCMV beamformer, described above, has an important limitation when spatially distinct yet temporally correlated sources are present in the EEG signal [10], [24]. Its main assumption is that the activity at the target location is not linearly correlated with activity at any other location. However, several studies of functional connectivity have suggested that temporal correlation relates to the communications among cortical areas. For example, such high correlations occur during evoked sensory responses in which the sensory information is transmitted to both left and right auditory cortices simultaneously, which result in almost perfectly correlated activities in the two hemispheres [41]. Correlated activities can also be observed in symmetric regions of the left and right hemispheres of the motor cortex [45], [46].

Different modifications of the single-core BF attempt to compensate for this limitation. The temporal correlation $M_{i, j}(f)$ of a pair of $(i, j)$ dipoles is quantified by the magnitude-squared cross spectrum $S_{i, j}(f)$ divided by the power spectra of both dipole moments $S_{i, i}(f)$ and $S_{j, j}(f)$

$$
M_{i, j}(f)=\frac{\left|S_{i, j}(f)\right|^{2}}{S_{i, i}(f) S_{j, j}(f)}
$$

The correlation is bounded between 0 and 1 , where $M_{i, j}(f)=1$ indicates a perfect linear relation between dipoles $d_{i}$ and $d_{j}$ at frequency $f$.

Dynamic imaging of coherent sources (DICS) is proposed in [9] where the spatial filter weighting matrix explicitly takes into account the estimated correlation quantified by (17). The authors of [47] conclude that high coherence results in a large error in the estimation of the dipole location. Low SNR additionally deteriorates the estimation of spatially close and temporally correlated dipoles. Correlated dipoles can be reliably localized if the distance between them is sufficiently high. DICS computes 
the cross spectral densities for any given location (from a dense grid of points) and all pair combinations of grid dipoles.

Inspired by the methodology of Diwakar et al. [26] we have developed an adaptive beamformer based on the LCMV algorithm with multiple constraints in the potentially correlated source locations. The optimization problem is solved using the method of Lagrange multipliers with multiple constraints

$$
\begin{aligned}
\min _{\boldsymbol{W}} & \operatorname{Tr}\left[\boldsymbol{W}^{t} \boldsymbol{C}_{v} \boldsymbol{W}\right] \\
\text { subject to } & \boldsymbol{W}^{t} \boldsymbol{L}\left(\boldsymbol{d}_{1}\right)=\boldsymbol{I} \\
& \boldsymbol{W}^{t} \boldsymbol{L}\left(\boldsymbol{d}_{2}\right)=\mathbf{0} \\
& \vdots \\
& \boldsymbol{W}^{t} \boldsymbol{L}\left(\boldsymbol{d}_{M}\right)=\mathbf{0} .
\end{aligned}
$$

The conventional beamformer is characterized with high computational costs due to the scanning solution over a 3-D source grid with thousands of nodes (potential source locations). The BF modifications to account for correlated sources increase even more the computational burden because of the additional cross correlation estimation for all pair combinations of grid dipoles. Moreover, the limited number of EEG channels restricts the degree of freedom and limits the number of constrains that can be considered.

We propose to deal with this problem by the iterative multicore BF-PF procedure where starting from randomly generated assumption for the dipole spatial coordinates of the active dipoles (uninformed prior) the PF converges to a small number of dominant sources. The multicore $\mathrm{BF}$ reconstructs the moments of each identified dominant source considering null constraints with respect to the others identified by the PF. The advantage of this combined solution is that the number of constrains in (18) is kept low and no a priori information for the expected spatial localization of the correlated sources is required. We rely on the estimation properties of the PF to converge to the actual active dipoles.

\section{Multicore BeAmformer-BAsed PARTicle Filter}

The multicore beamformer particle filter (multicore BPF) is a hybrid (statistical-deterministic) framework for reconstruction of correlated source. The PF provides estimates of the location of fixed temporally correlated brain sources in a 3-D space within the head defined by a grid of points. The ability to simultaneously and recursively estimate the source locations and waveforms lies in the BF spatial filter that is embedded within the particle filter framework to estimate the dipole moments for a given PF estimation of the dipole location. The multicore BPF algorithm is summarized below.

\section{Multicore Beamformer Particle Filter for Correlated Source} Localization

\section{1) Offline Computation}

Compute and store the forward matrices $\boldsymbol{L}$ for all points of the grid by solving the Maxwell equations in [33].

2) Initialization a) $k=0$, for $l=1, \ldots, N$, where $N$ denotes the total number of particles, generate samples $\boldsymbol{x}_{0}^{(l)} \sim p\left(\boldsymbol{x}_{0}\right)$ and set initial weights $\pi_{0}^{(l)}=1 / N$.

b) for $k=1,2, \ldots$.

3) Prediction Step

For $l=1, \ldots, N$, generate samples according to the state transition model in (12)

$$
\boldsymbol{x}_{k}^{(l)}=\boldsymbol{x}_{k-1}^{(l)}+\boldsymbol{w}_{k}^{(l)}, \text { where } \boldsymbol{w}_{k}^{(l)} \sim \mathcal{N}\left(\mathbf{0}, \sigma_{w}^{2} \boldsymbol{I}\right) .
$$

\section{4) Multicore Beamforming}

a) Find the lead field matrix $\boldsymbol{L}\left(\boldsymbol{x}_{k}^{(l)}\right)$ for each predicted dipole from the offline calculation.

b) Find the optimal spatial filter weights using (18). Consider the location of each estimated dipole $d_{i}(i=1, \ldots, M)$ as the targeted direction and the other $M-1$ dipoles as correlated with $d_{i}$ to compute the weighted vector associated with it.

c) Compute the source waveforms $\boldsymbol{s}_{k}^{(l)}$ according to (14).

\section{5) Measurement Update}

Evaluate the particle weights

a) For $l=1,2, \ldots, N$, on the receipt of a new measurement, compute the weights

$$
w_{k}^{(l)}=w_{k-1}^{(l)} \mathcal{L}\left(\boldsymbol{z}_{k} \mid\left(\boldsymbol{x}_{k}^{(l)}, \boldsymbol{L}\left(\boldsymbol{x}_{k}^{(l)}\right), \boldsymbol{s}_{k}^{(l)}\right)\right) .
$$

The likelihood $\mathcal{L}\left(\boldsymbol{z}_{k} \mid\left(\boldsymbol{x}_{k}^{(l)}, \boldsymbol{L}\left(\boldsymbol{x}_{k}^{(l)}\right), \boldsymbol{s}_{k}^{(l)}\right)\right)$ is calculated using (11).

b) For $l=1,2, \ldots, N$, normalize the weights

$$
\pi_{k}^{(l)}=w_{k}^{(l)} / \sum_{l=1}^{N} w_{k}^{(l)} .
$$

6) Evaluate the posterior mean as the estimate of the state at iteration $k$

$$
\hat{\boldsymbol{x}}_{k}=E\left[\boldsymbol{x}_{k} \mid \boldsymbol{Z}_{1: k}\right]=\sum_{l=1}^{N} \pi_{k}^{(l)} \boldsymbol{x}_{k}^{(l)} .
$$

7) Compute the effective sample size $N_{\mathrm{eff}}=$ $1 / \sum_{l=1}^{N}\left(\pi_{k}^{(l)}\right)^{2}$

8) Selection step (resampling) if $N_{\text {eff }}<N_{\text {thresh }}$ : multiply/suppress samples $\left\{\boldsymbol{x}_{k}^{(l)}\right\}$ with high/low weights $\pi_{k}^{(l)}$, in order to obtain $N$ new random samples approximately distributed according to the posterior state distribution.

Resampling is performed when the efficient number of particles $N_{\text {eff }}$ is below a fixed threshold $N_{\text {thresh }}$.

\section{Simulation Results}

The performance of the proposed approach is assessed by simulation experiments assuming the EEG signals are generated by a limited number of focal sources. A three-shell spherical head model (see Fig. 1) was created based on the following assumptions:

1) The head model consists of three concentric spherical shells with the enclosed space among them 

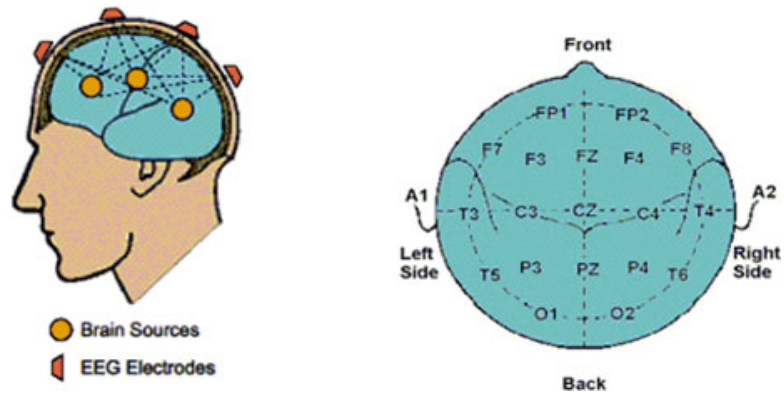

Fig. 1. Head model: (a) depiction of a realistic EEG experiment (left); (b) spatial scalp location of the EEG electrodes (right).

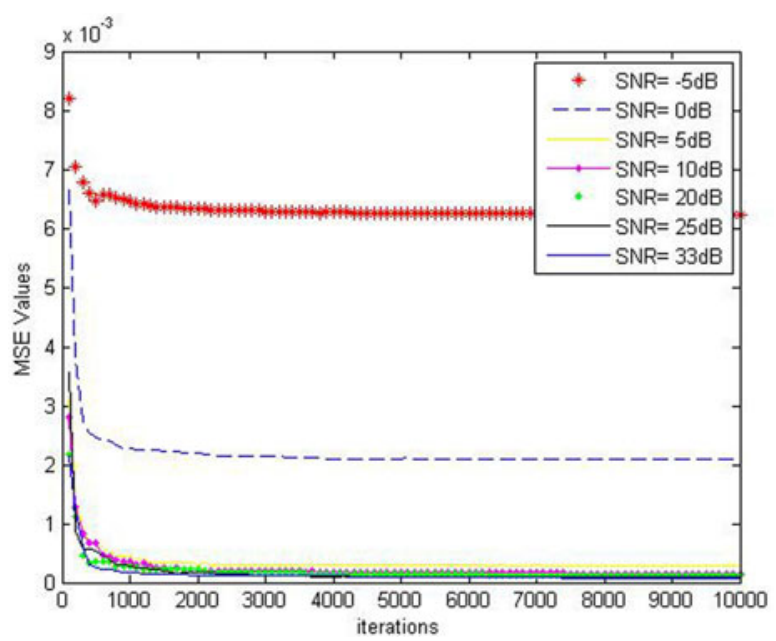

Fig. 2. Robustness of the proposed beamformer-based particle filter under varying SNR.

representing the scalp, skull and brain. The model dimensions are scaled to a realistic human head with an outer shell radius of $10 \mathrm{~cm}$, scalp radius of $9.2 \mathrm{~cm}$ and skull radius of $8.7 \mathrm{~cm}$.

2) Each layer is considered as homogeneous and isotropic, i.e., conductivity is constant and with no preferred direction. The conductivity values used for the head model were selected from studies on electrical impedance tomography aiming to create an electrical conductivity map of a volume [48]: scalp $0.33 \mathrm{~S} / \mathrm{m}$, skull $0.0165 \mathrm{~S} / \mathrm{m}$ and brain $0.33 \mathrm{~S} / \mathrm{m}$.

3) The distribution of the electrodes on the scalp follows the standard 10/20 International system with an array of 30electrodes: Fp1, AF3, F7, F3, FC1, FC5, C3, CP1, CP5, P7, P3, Pz, PO3, O1, Oz, O2, PO4, P4, P8, CP6, CP2, C4, FC6, FC2, F4, F8, AF4, Fp2, Fz, Cz.

4) The coordinates are defined with respect to a reference frame whose origin is located at the centre of the sphere: the $\mathrm{x}$-axis pointing in the direction of the right-ear, the $y$-axis pointing in the front of the head and the z-axis is taken to be vertical.

White noise was added into the generated EEG signals representing the effect of external sources not generated by brain activity, but by some disturbance (e.g., movements of muscles).
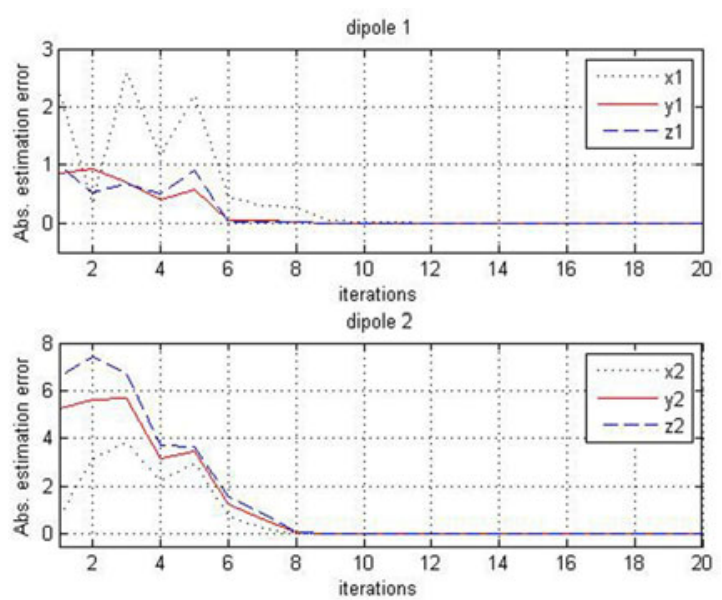

Fig. 3. Absolute estimation error of the dipole locations when the two brain sources are located on the dipole grid and SNR $=3 \mathrm{~dB}$.

The noise power was defined for different signal-to-noise ratios (SNR). The SNR is defined in the sensor domain as the total power of the signal divided by the total power of the noise added to the signal. The total searchable head volume is simulated with a fixed uniform grid model of 21012 points (potential dipoles). The leadfield matrix is computed off-line for each grid dipole. The experiments were done on a PC Intel Core with CPU 2 GHz, RAM 8 GB, 64-b OS, Windows 8.1.

\section{A. Dipole Localization Results}

Sinusoidal waveforms with amplitudes 0.1 and frequencies 10 and $15 \mathrm{~Hz}$ are assumed to be the brain signals originating from the two dipoles $\left(d_{1}\right.$ and $\left.d_{2}\right)$. Observe that the dimension of the state vector $\boldsymbol{x}_{k}=\left[x_{1 k}, y_{1 k}, z_{1 k}, x_{2 k}, y_{2 k}, z_{2 k}\right]^{t}$ is 6 , corresponding to three space coordinates per dipole. For the initial state vector, $\mathrm{N}=500$ samples are randomly generated from a uniform distribution in the interval $\boldsymbol{x}_{0} \in[\min (D), \max (D)]$ with $\boldsymbol{D}$ is the coordinates of a grid of dipoles, i.e., $\boldsymbol{D}=\left\{d_{i}=\right.$ $\left.\left[x_{i}, y_{i}, z_{i}\right]\right\}$.

The PF finds the brain source coordinates $\boldsymbol{x}_{k}$ within the head geometry as presented in Fig. 1.

In the simulations, the sources are randomly generated and, therefore, they may or may not coincide with the dipole grid that describes the head model. We consider three cases: 1) the two brain sources are located on the dipole grid; 2) only one brain source coincides with a dipole grid, and 3) none of the brain sources is located on the dipole grid. Figs. 3-5 show the absolute estimation error for the three cases with all simulations running for 200 iterations or time points. For display quality, we only show the first iterations after which the algorithm converges. We observe that the absolute estimation errors with respect to the space coordinates $(x, y, z)$ converge almost to zero after ten iterations if the original brain sources are located on the grid head model. The ground truth dipoles are $d_{1}:(0.0116,0.0767,0.019) \mathrm{m}$ and $d_{2}:(-0.0116,-0.0767,0.0095)$ $\mathrm{m}$.

The estimation of the locations of nongrid-dipoles ends with a small steady-state error. The ground truth dipoles are now close 


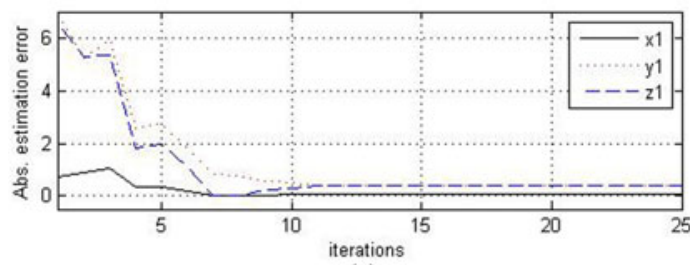

(a)

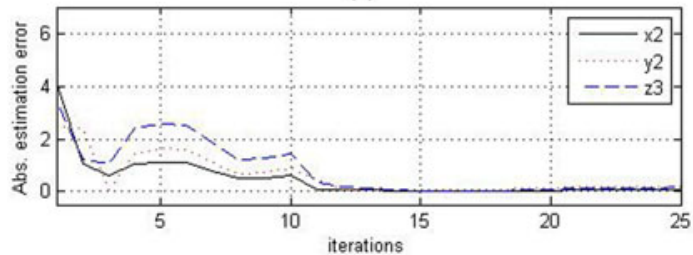

(b)

Fig. 4. Absolute estimation error of the dipole locations when only the second brain source coincides with a dipole grid and $\mathrm{SNR}=3 \mathrm{~dB}$.
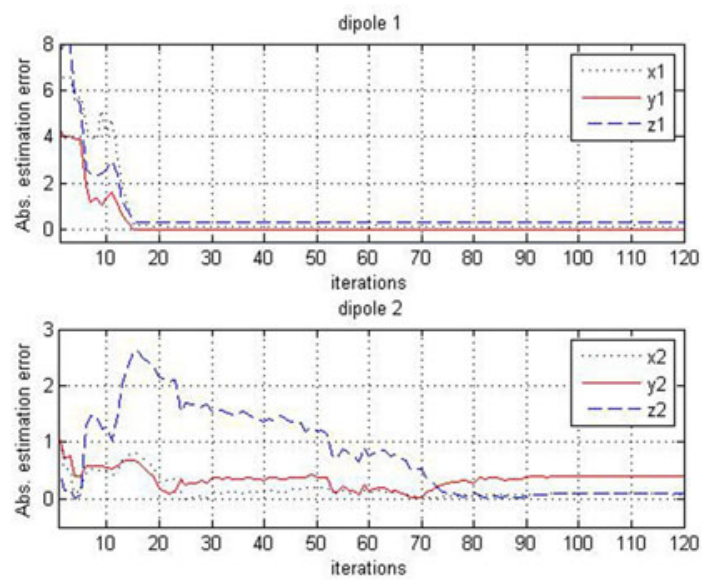

Fig. 5. Absolute estimation error of the dipole locations when none of the brain sources is located on the dipole grid and $\mathrm{SNR}=3 \mathrm{~dB}$.

but do not coincide with any grid point $d_{1}:(0.01,0.075,0.02) \mathrm{m}$ and $d_{2}:(-0.01,-0.075,-0.01) \mathrm{m}$. This error can be reduced if the grid model is more dense. However, including very closely spaced sources lead to ill-conditioned null-constrains in [28], [49]. It is worth pointing out that in Figs. 3-5 the differences in the convergence behavior between the two dipoles are mainly due to the small number of particles $(N=500)$. If the number of particles increases enough, the convergence behavior of the two dipoles (in every scenario) would be statistically similar.

The robustness of the proposed multicore BPF to the noise in the EEG dataset was also studied. Specifically, we generated EEG data with different noise powers according to (9). Fig. 2 shows the spatial mean-square error (MSE), for different SNR, computed as follows:

$$
\operatorname{MSE}=\left(\sqrt{(\hat{x}-x)^{2}+(\hat{y}-y)^{2}+(\hat{z}-z)^{2}}\right)
$$

where $(x, y, z)$ are the ground truth coordinates and $(\hat{x}, \hat{y}, \hat{z})$ are the estimated positions. We observe that, as long as the signal power is higher than the noise power (SNR $>0 \mathrm{~dB}$ ), the MSE
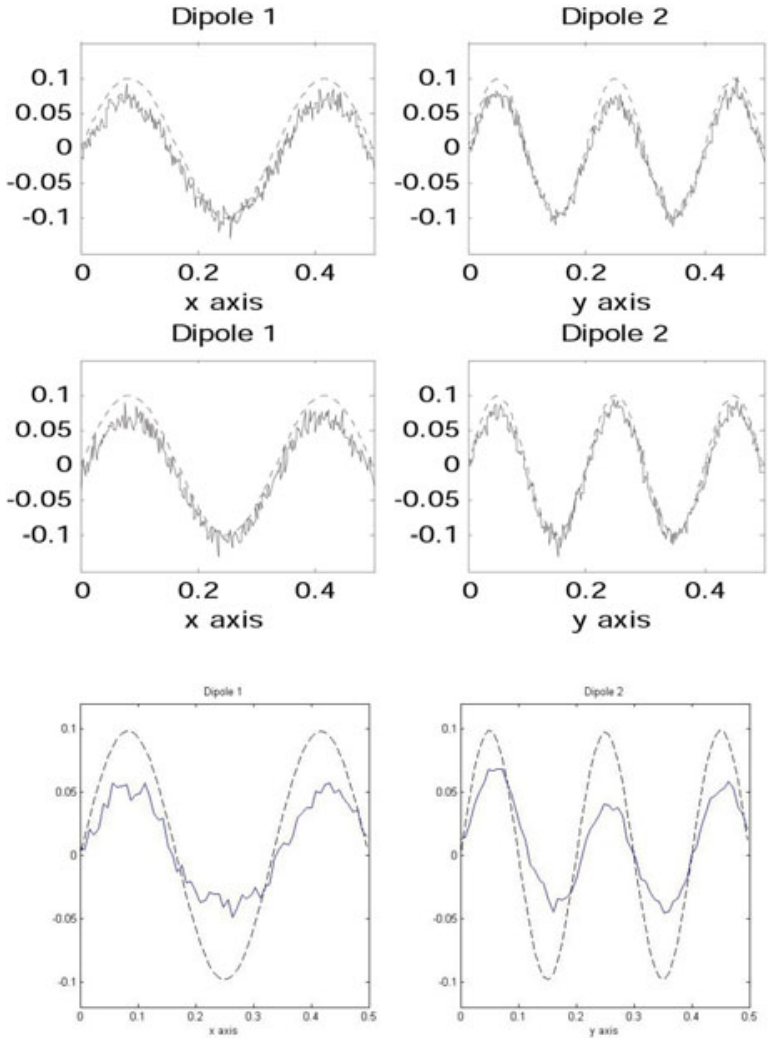

Fig. 6. Source waveform estimation by beamforming for uncorrelated dipoles: the original (dotted line) and the estimated curve (bold line) for dipole 1 (left) and dipole 2 (right) using the multicore $\mathrm{BF}$ (top plots), the single-core $\mathrm{BF}$ (middle plots) and the full PF (bottom plots) with SNR $=3 \mathrm{~dB}$.

converges close to zero steady-state error. MSE degrades for EEG corrupted with severe noise $(\mathrm{SNR} \leq 0 \mathrm{~dB})$.

\section{B. Multicore BPF Versus Single-Core BPF and Full PF}

In order to validate the multicore BPF, we compare it with the two alternative techniques, single-core BPF and the full PF, from which the proposed method originated. The experiments were performed with the following control conditions: the neural activity from a-pair of correlated dipole sources with $95 \%(M=0.95)$ and $30 \%$ correlation $(M=0.3)$ were simulated as sinusoidal base waves with amplitudes 0.1 and frequencies 3 and $5 \mathrm{~Hz}$ over $0.5 \mathrm{~s}$. The performance was evaluated at low SNRs (3 and $8 \mathrm{~dB}$ ). The target dipoles (ground truth) were taken from the predefined grid with the following $(x, y, z)$ coordinates: $d_{1}:(0.01,0.075,0.02) \mathrm{m}$ (right frontal cortex) and $d_{2}:(-0.01,-0.075,-0.01) \mathrm{m}$ (left occipital cortex), with a dominant direction of propagation along the $\mathrm{x}$-axis for $d_{1}$ and along the y-axis for $d_{2}$ defined by the following vectors: $\operatorname{dir}_{1}:(0.8,0.1,0.1)$ and $\operatorname{dir}_{2}:(0.1,0.8,0.1)$. The rational behind this choice is to generate sources located on opposite brain hemispheres; and thus they are spatially distinct but temporally correlated.

First, the effect of the dipole correlation (expressed by $M$ ) on the beamformer was evaluated (see Figs. 6-8). Note that the simulation of dipole correlation changes the sine shape of the 

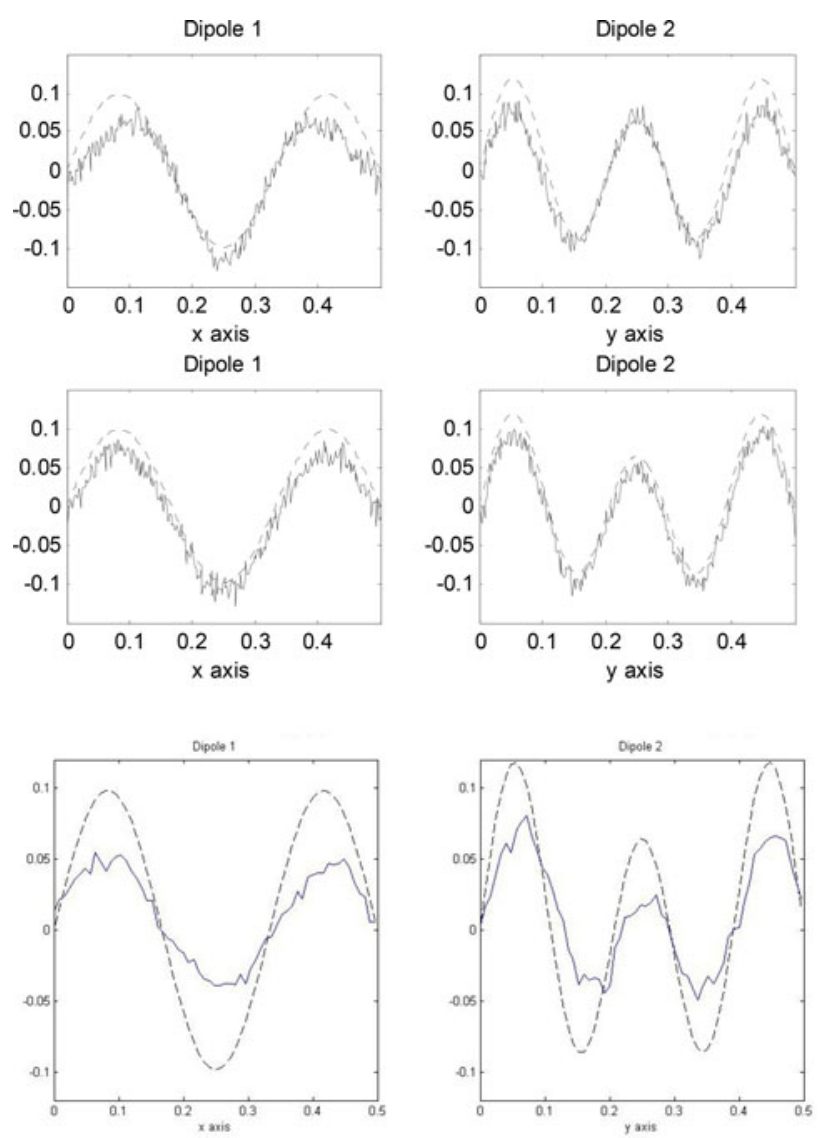

Fig. 7. Source waveform estimation by beamforming for $M=0.3$ (low correlation): the original (dotted line) and the estimated curve (bold line) for dipole 1 (left) and dipole 2 (right) using the multicore BF (top plots), the single-core $\mathrm{BF}$ (middle plots) and the full PF (bottom plots) with SNR $=3 \mathrm{~dB}$.

base signal. The single-core BF and the multicore BF provide very similar estimations for uncorrelated dipoles. The higher the correlation level $(M=0.95)$, the more biased are the estimations of the single-core BF as can be seen in Fig. 8. This is due to the filter weight matrix that was computed assuming the source time-courses come from uncorrelated generators.

The results of the spatial location estimation by the three methods are depicted in Fig. 10 for $M=0.3$ (low correlation) and in Fig. 11 for $M=0.95$ (high correlation). For low correlation levels (relatively independent sources) the estimations of the three methods are very similar. The multicore BPF clearly outperforms the other methods in the case of highly correlated dipoles. Figs. 10 and 11 partially show the volume dipole grid over which the particle filter conducts the search.

Table I summarizes the spatial MSE under varying SNR and varying correlation levels $M$ for 500 particles, 200 time steps, across ten Monte Carlo simulations. Even from very noisy EEG data (SNR $=3 \mathrm{~dB}$ ) and without any prior assumption about the true location of the dipoles, the multicore BPF provides estimation within 3-5 mm error distance. The single-core BPF can achieve competitive accuracy, but only for dipoles with low or none temporal correlation. The full PF is less sensitive to dipole correlation and noise. The PF estimation error is relatively
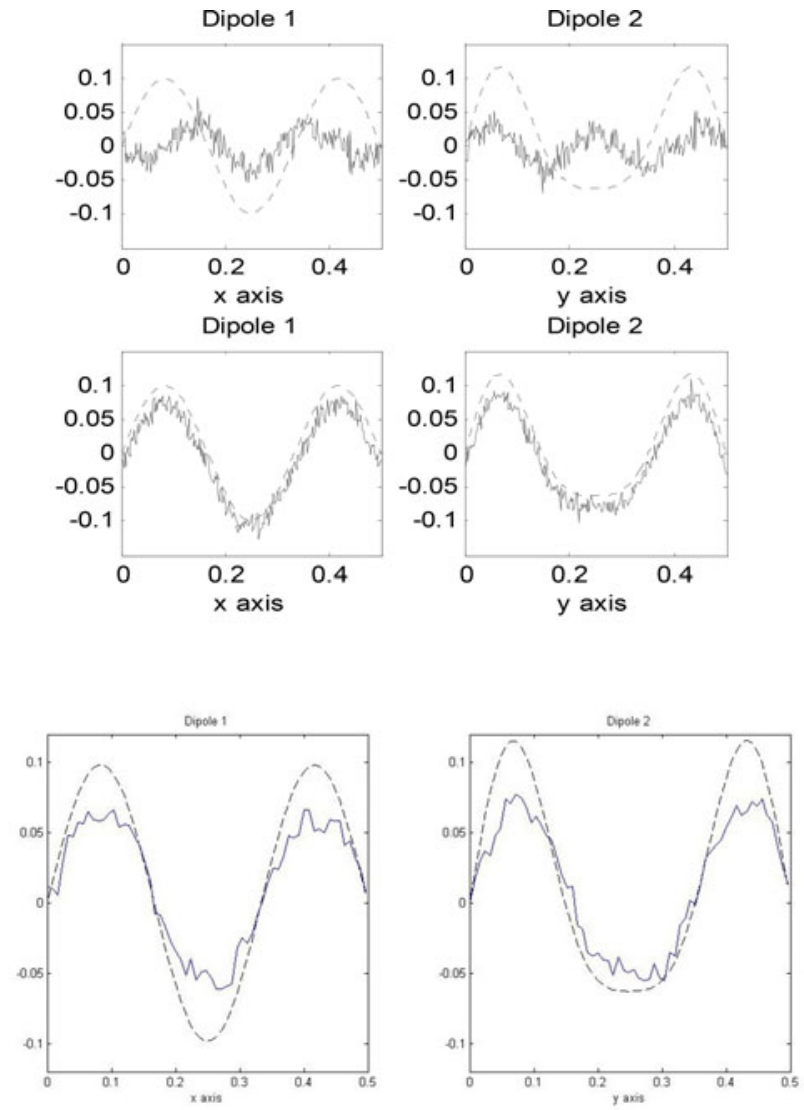

Fig. 8. Source waveform estimation by beamforming for $M=0.9$ (high correlation): the original (dotted line) and the estimated curve (bold line) for dipole 1 (left) and dipole 2 (right) using the multicore BF (top plots), the singlecore $\mathrm{BF}$ (middle plots) and the full PF (bottom plots) with SNR $=3 \mathrm{~dB}$.
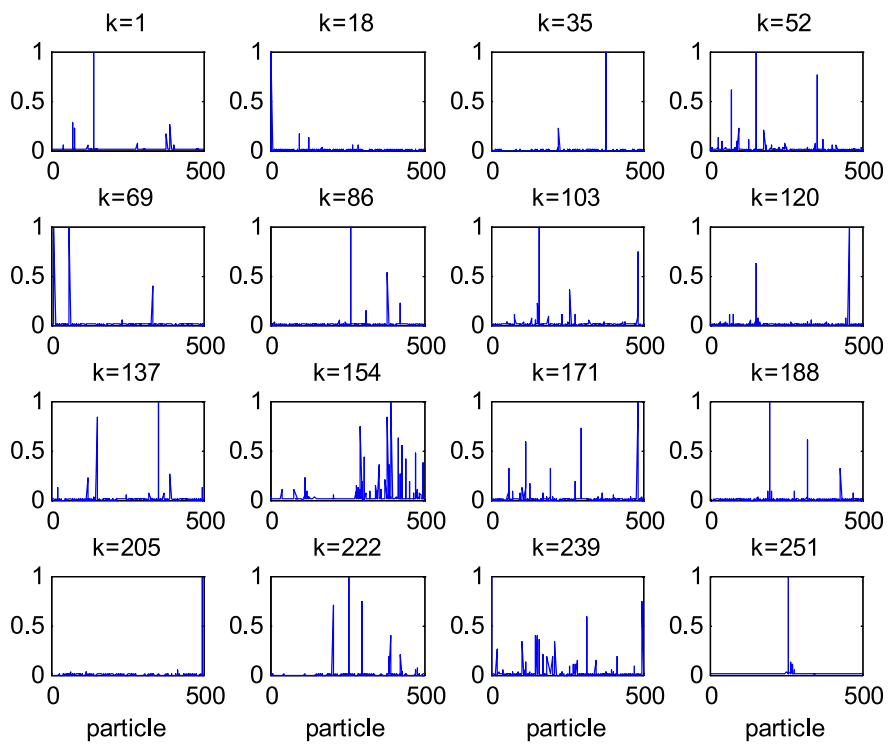

Fig. 9. Normalized weights [see (21)] computed over the recursive PF estimation. 


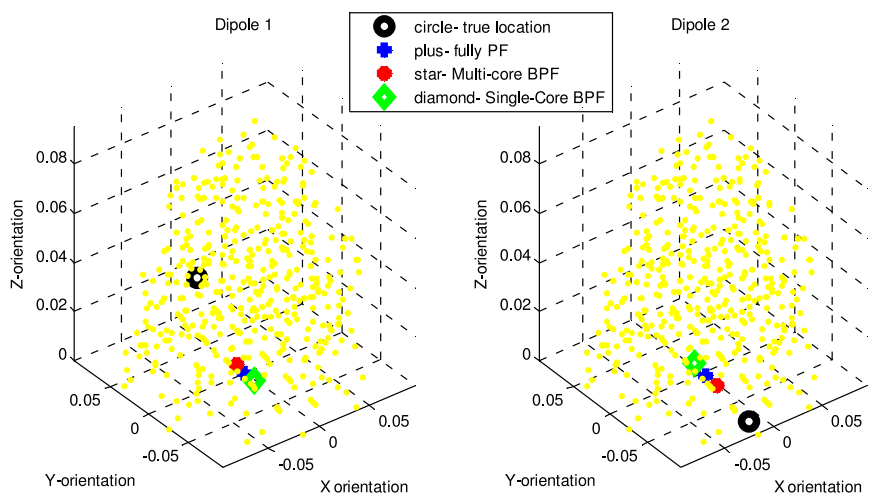

Fig. 10. Spatial location estimates of the dipoles and the ground truth (black) for low correlation, $M=0.3, \mathrm{SNR}=3 \mathrm{~dB}$.

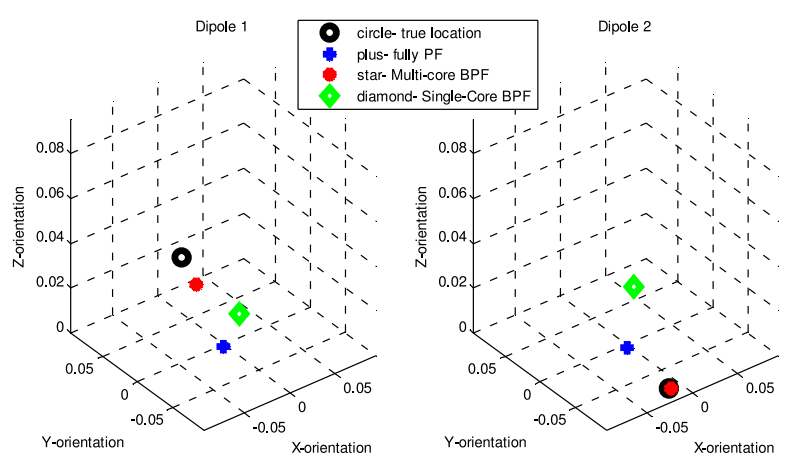

Fig. 11. Spatial location estimates of the dipoles and the ground truth (black) for high correlation, $M=0.95, \mathrm{SNR}=3 \mathrm{~dB}$.

high, however if the number of the particles is higher (only 500 in the present scenario) it has the potential to recover better the dipole location. However, a significant amount of memory and computational power are needed, especially when the number of estimated dipoles increases.

Fig. 9 presents the normalized weights computed over the recursive PF estimation for some of the iterations $k$. Note that based on the current likelihood value at each iteration only few of the particles (from $N=500$ particles in total) are pointed out as the most probable candidates for the location of the dipoles. This reduces significantly the computational efforts associated with the exhaustive search over the complete dipole grid conducted by the full beamforming approach or other deterministic parametric methods for brain source localization. In addition, the computational gain of the proposed BPF is exponential as compared to the full PF. The main computational burden, both in the hybrid approach and in the full PF approach, comes from the PF. The power of the PF in handling nonlinear systems comes at a computational cost. The approximation error of the PF grows exponentially in the dimension of the state vector. It has been shown that the PF collapses unless the number of particles grows superexponentially in the system dimension [50]. This phenomenon has rendered the PF of limited use in highdimensional problems. In the proposed BPF approach, the PF estimates the location of the dipoles, whereas the beamformer estimates the dipole waveforms. In the full PF algorithm, both the location and the waveform are estimated using the PF. In particular, the dimension of the state vector in the full PF framework is double the dimension of the state vector in the BPF approach. In our preliminary simulations (not shown here for space limitations), we found that the full PF is able to converge to a near-zero error for one dipole. However, for two or more dipoles, the full PF converges to a nonzero error that increases as the number of dipoles increases (i.e., the number of the state dimension increases). These results are in accordance with the known "curse of dimensionality" issue in particle filtering. By reducing the dimension of the state vector that must be estimated by the PF, the proposed beamformer-PF exponentially reduces the computational burden of the problem.

The computer simulations demonstrate the efficiency of the proposed method for localizing and reconstructing highly correlated sources brain sources from noisy EEG data. A spherical model that approximate the head by three concentric spherical shells representing the brain, skull and scalp is used as in most of the references cited. However, this is a simplification because knowledge of the electrical conductivity map of the head is important since it is known that the solution to the source localization problem is highly dependent on the values taken by the scalp, skull, and brain conductivities [51]. Realistic head modeling of both geometry and anisotropy can further improve the performance of the beamformer for low SNR.

\section{Results on Real EEG Data}

In this section, we demonstrate the estimation accuracy of the proposed algorithm with real EEG data. The data corresponds to visually evoked potential (VEP) signals extracted from thirteen female subjects (20-28 years old). All participants had normal or corrected to normal vision and no history of neurological or psychiatric illness. Different facial expressions (neutral, fearful and disgusted) of 16 individuals (eight males and eight females) were selected, giving a total of 48 different facial stimuli. Images of 16 different house fronts were superimposed on each of the faces. This resulted in a total of 384 grayscale composite images (9.5 cm wide by $14 \mathrm{~cm}$ high) of transparently superimposed face and house.

Participants were seated in a dimly lit room, where a computer screen was placed at a viewing distance of approximately $80 \mathrm{~cm}$ coupled to a PC equipped with software for the EEG recording. The images were divided into two experimental blocks. In the first, the participants were required to attend to the houses (ignoring the faces) and in the other they were required to attend to the faces (ignoring the houses). The participants task was to determine, on each trial, if the current house or face (depending on the experimental block) is the same as the one presented on the previous trial. Stimuli were presented in a sequence of $300 \mathrm{~ms}$ each and were preceded by a fixation cross displayed for $500 \mathrm{~ms}$. The intertrial interval was $2000 \mathrm{~ms}$.

EEG signals were recorded from 20 electrodes (Fp1, Fp2, F3, F4, C3, C4, P3, P4, O1, O2; F7, F8, T3, T6; P7, P8, Fz, Cz, Pz, $\mathrm{Oz}$ ) according to the 10/20 International system. Electrooculogram (EOG) signals were also recorded from electrodes placed just above the left supra orbital ridge (vertical EOG) and on the 
This article has been accepted for publication in a future issue of this journal, but has not been fully edited. Content may change prior to final publication. Citation information: DOI 10.1109/JBHI.2015.2413752, IEEE Journal of Biomedical and Health Informatics

TABLE I

Spatial MSE in Millimeters Under VARYing SNR ANd CoRrelation LeVEls $M$ For $N=500$ Particles AND 200 Iterations

\begin{tabular}{lccccccccc}
\hline \hline Method & \multicolumn{4}{c}{$\mathrm{SNR}=3 \mathrm{~dB}$} & & \multicolumn{2}{c}{$\mathrm{SNR}=8 \mathrm{~dB}$} \\
& \multicolumn{2}{c}{ Dipole 1 } & \multicolumn{2}{c}{ Dipole 2 } & & Dipole 1 & & Dipole 2 \\
\hline & $M=0.95$ & $M=0.3$ & $M=0.95$ & $M=0.3$ & $M=0.95$ & $M=0.3$ & $M=0.95$ & $M=0.3$ \\
Full PF & 8.2 & 8.3 & 7.3 & 7.6 & 6.9 & 6.7 & 5.8 & 5.3 \\
Single-Core BPF & 12.2 & 3.95 & 9.97 & 3.3 & 11.5 & 3.3 & 8.7 & 3.1 \\
Multicore BPF & 3.4 & 5.42 & 1.8 & 4.41 & 2.8 & 4.1 & 1.5 & 3.6 \\
\hline
\end{tabular}

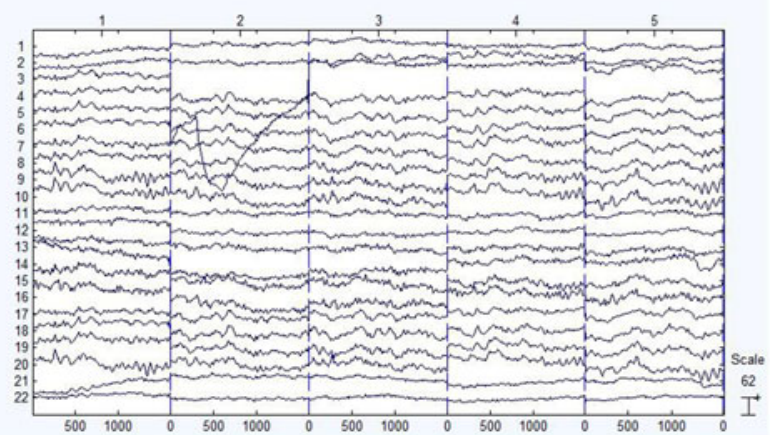

Fig. 12. EEG signals (channels 1-20) and EOG signals (channels 21-22). Horizontal axis [ms], vertical axis [EEG channels].

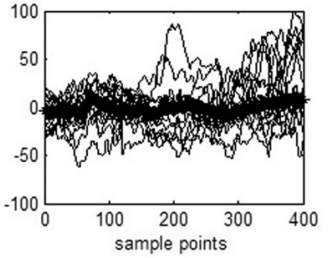

(a)

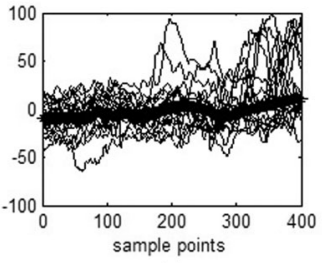

(c)

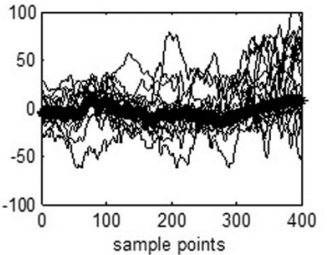

(b)

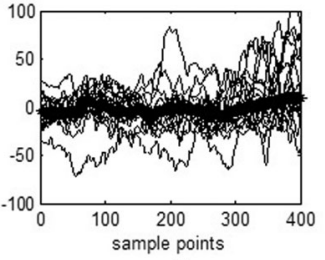

(d)
Fig. 13. Superposition of 18 PCA enhanced VEP recorded by four electrodes: (a) $\mathrm{O} 1$; (b) O2; (c) Pz; (d) Oz. The bold trace represents the average of all trials used to test the particle filter.

left outer canthus (horizontal EOG). VEP were calculated offline averaging segments of 400 points of digitized EEG (12-b A/D converter, sampling rate $250 \mathrm{~Hz}$ ). These segments covered 1600 ms comprising a prestimulus interval of $148 \mathrm{~ms}$ (37 samples) and poststimulus onset interval of $1452 \mathrm{~ms}$. The EEG signal was visually inspected, prior to processing, and those segments with excessive EOG artifacts were manually eliminated (see Fig. 12 where epoch 2 was manually discarded). Only trials with correct responses were included in the data set. The experimental setup was designed by Santos et al. [52] for their study on subject attention and perception using VEP signals.

Fig. 13 represents 18 trials of four channels enhanced by principal component analysis (PCA). In the reconstructed signals,
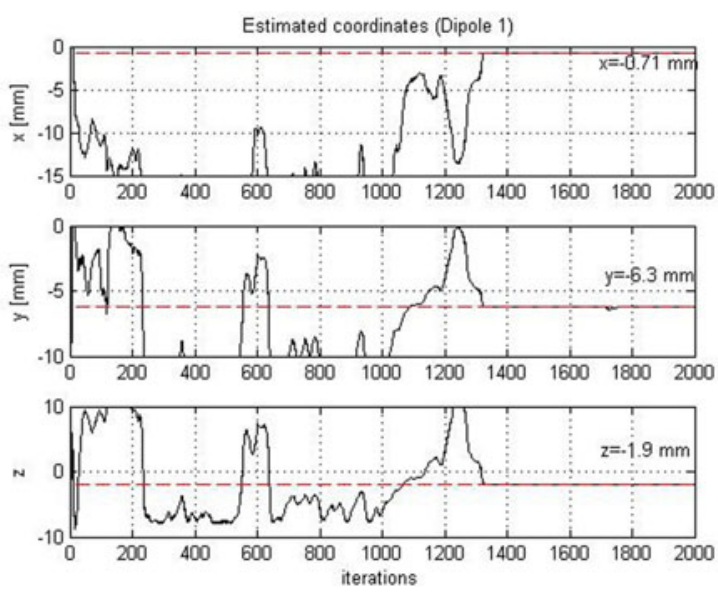

Fig. 14. Estimation of the source location (Dipole 1) that produced the P100 peak.
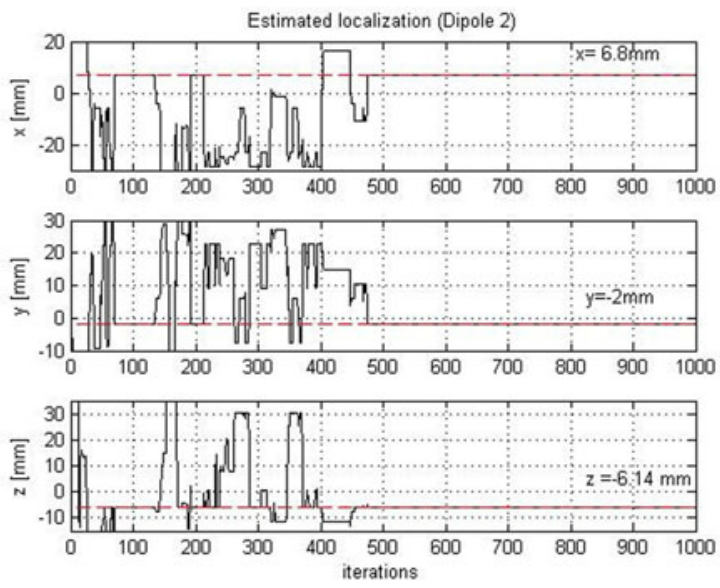

Fig. 15. Estimation of the source location (Dipole 2) that produced the P100 peak.

it is possible to identify a positive peak in the range of 100-160 ms, known as P100. P100 corresponds to the perception of the sensory stimulus, a brain activity that is known to happen in the primary visual cortex. The occipital channels $(\mathrm{O} 1, \mathrm{Oz})$ that measure the brain activity around the visual cortex present the largest peak. We apply the proposed BPF to estimate the two strongest sources $\left(d_{1}\right.$ and $\left.d_{2}\right)$ that may have produced the P100 peak. The results of the estimation are summarized in Figs. 14 and 15. The dipole reconstruction from real EEG data took more iterations than with synthetic data, about 1300 iterations for dipole 1 and 480 iterations for dipole 2. After that the PF weights 


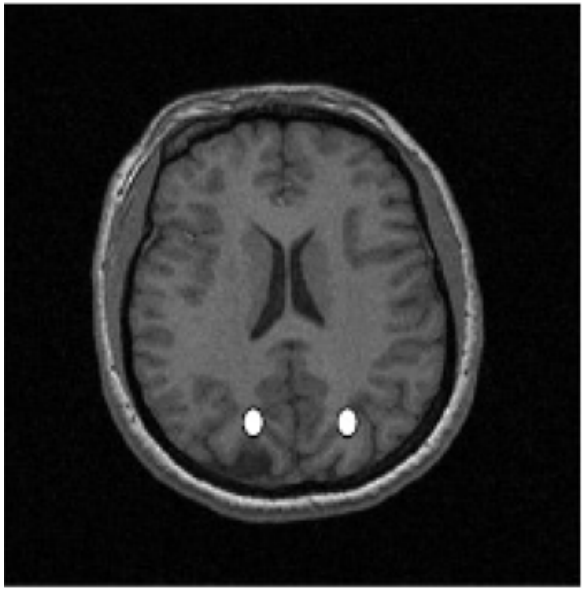

Fig. 16. Primary visual cortex: axial view. The estimated active zones are depicted in white circles.

converged to fixed values and therefore the identified spatial coordinates reached steady states. It is very interesting to observe that the final coordinates of $d_{1}:(0.71 \mathrm{~mm},-6.3 \mathrm{~mm},-1.9 \mathrm{~mm})$ and $d_{2}:(6.8 \mathrm{~mm},-2 \mathrm{~mm},-6.14 \mathrm{~mm})$ correspond to the zone of the primary visual cortex as illustrated in Fig. 16. Therefore, the proposed beamformer-based PF successfully estimated the space coordinates of the two strongest brain sources, producing the P100 peak, as located in the zone of the primary visual cortex.

\section{CONCLUSIONS}

This paper proposes a multicore beamformer particle filter (multicore BPF) for solving the ill-posed EEG inverse problem. The method combines a particle filter (statistical approach) for estimation of the spatial location and a multicore beamformer (deterministic approach) for estimation of temporally correlated dipole waveforms in a recursive framework. As a result the estimation accuracy is improved. This general framework comprising the multicore BF allows to cope with the main challenges of the EEG brain source recovering with particular emphases upon temporally correlated dipoles. We conducted extensive simulations, based on generated and real EEG experiments, in order to study the accuracy and robustness of the proposed algorithm. The multicore BPF guarantees convergence to the correct spatial-temporal source coordinates as long as the power of the signal is higher than the power of the noise within the EEG measurements. We have also conducted EEG experiments where subjects were exposed to visual stimuli. The multicore BPF localized the two strongest brain sources that have produced the recorded EEG signal within the expected visual cortex zone. Numerous challenges still remain for an objective assessment of the relative performance of inverse algorithms and the statistical significance of different solutions computed from simulated and experimental data. Additional research efforts are needed to come up with a real-time solution of the inverse problem. Our recent ongoing study suggests that the proposed methodology can be transferred from the "fixed dipoles" case (as in the present study) to the "moving dipoles" case with encouraging results.

\section{ACKNOWLEDGMENT}

The authors would like to thank B. Ebinger, a graduate student with the Department of Electrical and Computer Engineering, Rowan University, for his contribution to the implementation of some of the code.

\section{REFERENCES}

[1] S. Sanei and J. Chambers, EEG Signal Processing. New York, NY, USA: Wiley, 2007

[2] A. Galka, O. Yamashita, T. Ozaki, R. Biscay, and P. Valds-Sosa, "A solution to the dynamical inverse problem of EEG generation using spatiotemporal Kalman filtering," Neuroimage, vol. 23, no. 2, pp. 435-453, 2004.

[3] B. Kamousi, Z. Liu, and B. He, "An EEG inverse solution based braincomputer interface," Int. J. Bioelectromagn., vol. 7, no. 2, pp. 292-294, 2005.

[4] L. Qin, L. Ding, and B. He, "Motor imagery classification by means of source analysis for brain computer interface applications," J. Neural Eng., vol. 2, no. 4, pp. 65-72, Dec. 2005.

[5] Q. Noirhomme, R. Kitney, and B. Macq, "Single-trial EEG source reconstruction for brain-computer interface," IEEE Trans. Biomed. Eng., vol. 55, no. 5, pp. 1592-1601, May 2008.

[6] M. Grosse-Wentrup, C. Liefhold, K. Gramann, and M. Buss, "Beamforming in non-invasive brain-computer interfaces," IEEE Trans. Biomed. Eng., vol. 56, no. 4, pp. 1209-1219, Apr. 2009.

[7] S. Baillet, J. Mosher, and R. Leahy, "Electromagnetic brain mapping," IEEE Signal Process. Mag., vol. 18, no. 6, pp. 14-30, Nov. 2001.

[8] C. Michel, M. Murray, G. Lantz, S. Gonzalez, L. Spinelli, and R. G. de Peralta, "EEG source imaging," Clin. Neurophysiol., vol. 115, no. 10 , pp. 2195-222, Oct. 2004.

[9] J. Gross and A. Ioannides, "Linear transformations of data space in meg," Phys. Med. Biol., vol. 44, no. 8, pp. 2081-2097, Aug. 1999.

[10] B. V. Veen, W. V. Drongelen, M. Yuchtman, and A. Suzuki, "Localization of brain electrical activity via linearly constrained minimum variance spatial filter," IEEE Trans. Biomed. Eng., vol. 44, no. 9, pp. 867-880, Sep. 1997.

[11] A. Cichocki and S. Amari, Adaptive Blind Signal and Image Processing: Learning Algorithms and Applicaions. New York, NY, USA: Wiley, Jul. 2002.

[12] A. Hyvärinen, J. Karhunen, and E. Oja, Independent Component Analysis New York, NY, USA: Wiley, 2001.

[13] S. J. Kiebel, J. Daunizeau, C. Phillips, and K. J. Friston, "Variational Bayesian inversion of the equivalent current dipole model in EEG/MEG," NeuroImage, vol. 39, no. 2, pp. 728-741, Jan. 2008.

[14] A. Sorrentino, L. Parkkonen, and M. Piana, "Particle filters: A new method for reconstructing multiple current dipoles from MEG data," Int. Congr Series, vol. 1300, pp. 173-176, 2007.

[15] A. Sorrentino, L. Parkkonen, A. Pascarella, C. Campi, and M. Piana, "Dynamical MEG source modeling with multi-target Bayesian filtering," Human Brain Mapping, vol. 30, no. 6, pp. 1911-1921, Jun. 2009.

[16] M. Arulampalam, S. Maskell, N. Gordon, and T. Clapp, "A tutorial on particle filters for online nonlinear/non-Gaussian Bayesian tracking," IEEE Trans. Signal Process., vol. 50, no. 2, pp. 174-188, Feb. 2002.

[17] A. Doucet and A. M. Johansen, "A tutorial on particle filtering and smoothing: Fifteen years later," in Handbook of Nonlinear Filtering. Oxford, U.K.: Oxford Univ. Press, 2009, pp. 656-704.

[18] F. Gustafsson, "Particle filter theory and practice with positioning applications," IEEE Trans. Aerospace Electron. Syst. Mag. Part II: Tuts. vol. 25, no. 7, pp. 53-82, Jul. 2010.

[19] L. Mihaylova, A. Y. Carmi, F. Septier, A. Gning, S. K. Pang, and S. Godsill, "Overview of Bayesian sequential Monte Carlo methods for group and extended object tracking," Digital Signal Process., vol. 25, no. 1, pp. 1-16, 2014.

[20] O. Cappé, S. Godsill, and E. Mouline, "An overview of existing methods and recent advances in sequential Monte Carlo," IEEE Proc., vol. 95, no. 5, pp. 899-924, May 2007.

[21] J. Antelis and J.Minguez, "Dynamic solution to the EEG source localization problem using Kalman filters and particle filters," in Proc. Int. Conf. IEEE Eng. Med. Biol. Soc., 2009, pp. 77-80.

[22] H. Mohseni, E. Wilding, and S. Sanei, "Sequential Monte Carlo techniques for EEG dipole placing and tracking," in Proc. 5th IEEE Sensor Array Multichannel Signal Process. Workshop, Jul. 2008, pp. 95-98. 
[23] L. Miao, J. Zhang, C. Chakrabarti, and A. Papandreou-Suppappola, "Efficient Bayesian tracking of multiple sources of neural activity: Algorithms and real-time FPGA implementation," IEEE Trans. Signal Process., vol. 61, no. 3, pp. 633-647, Feb. 2013.

[24] K. Sekihara, S. Nagarajan, D. Poeppel, A. Marantz, and Y. Miyashita, "Reconstructing spatio-temporal activities of neural sources using an MEG vector beamformer technique," IEEE Trans. Biomed. Eng., vol. 48, no. 7, pp. 760-771, Jul. 2001.

[25] M. Brookes, C. Stevenson, G. Barnes, A. Hillebrand, M. Simpson, S. Francis, and P. Morris, "Beamformer reconstruction of correlated sources using a modified source model," Neuroimage, vol. 34, pp. 1454$1465,2007$.

[26] M. Diwakar, M.-X. Huang, R. Srinivasan, D. Harrington, A. Robb, A. Angeles, L. Muzzatti, R. Pakdaman, T. Song, R. Theilmann, and R. Lee, "Dual-core beamformer for obtaining highly correlated neural networks in MEG," Neuroimage, vol. 54, pp. 253-263, 2011.

[27] S. Dalal, K. Sekihara, and S. S. Nagarajan, "Modified beamformers for coherent source region suppression," IEEE Trans. Biomed. Eng., vol. 53, no. 7, pp. 1357-1363, Jul. 2006.

[28] M. Popescu, E. Popescu, T. Chan, S. Blunt, and J. D. Lewine, "Spatiotemporal reconstruction of bilateral auditory steady-state responses using MEG beamformers," IEEE Trans. Biomed. Eng., vol. 55, no. 3, pp. 10921102, Mar. 2008.

[29] J. Mosher and R. Leahy, "Recursive music: a framework for EEG and MEG source localization," IEEE Trans. Biomed. Eng., vol. 45, no. 11, pp. 1342-1354, Nov. 1998.

[30] N. J. Gordon, D. J. Salmond, and A. F. M. Smith, "Novel approach to nonlinear/non-Gaussian Bayesian state estimation," IEE Proc., vol. 140, no. 2, pp. 107-113, Apr. 1993.

[31] A. Doucet, N. Freitas, and E. N. Gordon, Sequential Monte Carlo Methods in Practice. New York, NY, USA: Springer, 2001.

[32] S. Haykin, Adaptive Filter Theory. Englewood Cliffs, NJ, USA: Prentice Hall, 2001.

[33] Y. Salu, L. Cohen, D. Rose, S. Sato, C. Kufta, and M. Hallet, "An improved method for localizing electric brain dipoles," IEEE Trans. Biomed. Eng., vol. 37, no. 7, pp. 699-705, Jul. 1990.

[34] B. Anderson and J. Moore, Optimal Filtering. New York, NY, USA: Dover, 2005.

[35] B. Ristic, S. Arulampalam, and N. Gordon, Beyond the Kalman Filter: Particle Filters for Tracking Applications. Norwood, MA, USA: Artech House, 2004.

[36] T. R. Knösche, E. M. Berends, H. R. Jagers, and M. J. Peters, "Determining the number of independent sources of the EEG: A simulation study on information criteria," Brain Topography, vol. 11, no. 2, pp. 111-124, 1998.

[37] S. Robinson and J. Vrba, "Functional neuroimaging by synthetic aperture magnetometry (SAM)," in Recent Advances in Biomagnetism. Sendai, Japan: Tohoku Univ. Press, 1999, pp. 302-305.

[38] A. Hillebrand and G. Barnes, "The use of anatomical constraints with MEG beamformers," Neuroimage, vol. 20, no. 4, pp. 2302-2313, 2003.

[39] M. Huang, J. Shih, R. Lee, D. Harrington, R. Thoma, M. Weisend, F. Hanion, K. Paulson, T. Li, K. Martin, G. Miller, and J. Canive, "Commonalities and differences among vectorized beamformers in electromagnetic source imaging," Brain Topography, vol. 16, no. 3, pp. 139-158, 2004.

[40] C. Chen, A. Pogosyan, L. Zrinzo, S. Tisch, P. Limousin, K. Ashkan, T. Yousry, M. Hariz, and P. Brown, "Intra-operative recordings of local field potentials can help localize the subthalamic nucleus in Parkinson's disease surgery," Exp. Neurology, vol. 198, pp. 214-221, 2006.

[41] A. Herdman, A. Wollbrink, W. Chan, R. Ishii, B. Ross, and C. Pantev, "Determination of activation areas in the human auditory cortex by means of synthetic aperture magnetometry," Neuroimage, vol. 20, no. 2, pp. 995$1005,2003$.

[42] D. Cheyne, A. Bostan, W. Gaetz, and E. Pang, "Event-related beamforming: a robust method for presurgical functional mapping using MEG," Clin. Neurophysiol., vol. 118, no. 8, pp. 1691-1704, 2007.

[43] H. Yuan, A. Doud, A. Gururajan, and B. He, "Cortical imaging of eventrelated (de)synchronization during online control of brain-computer interface using minimum-norm estimates in frequency domain," IEEE Trans. Neural Syst. Rehabil. Eng., vol. 16, no. 5, pp. 425-431, Oct. 2008.

[44] J. Vrba and S. E. Robinson, "Signal processing in magnetoencephalography," Methods, vol. 25, no. 2, pp. 249-271, 2001.

[45] E. Gysels, P. Renevey, and P. Celka, "SVM-based recursive feature elimination to compare phase synchronization computed from broadband and narrowband EEG signals in brain computer interfaces," Signal Process., vol. 85 , no. 11, pp. 2178-2189, 2005.
[46] Y. Lai, W. V. Drongelen, L. Ding, K. Hecox, V. Towle, D. Frim, and B. He, "Estimation of in vivo human brain-skull conductivity ratio from simultaneous extra and intra-cranial electrical potential recordings," Clin. Neurophysiol., vol. 116, no. 2, pp. 456-465, Feb. 2005.

[47] H. Yuan, T. Liu, R. Szarkowski, C. R. J. Ashe, and B. He, "An EEG and fMRI study of motor imagery: Negative correlation of bold and EEG activity in primary motor cortex," Neuroimage, vol. 49, pp. 2596-2606, 2010

[48] O. Gilad, L. Horesh, and D. Holder, "Design of electrodes and current limits for low frequency electrical impedance tomography of the brain," Med. Biomed. Eng. Comput., vol. 45, no. 7, pp. 621-633, Jul. 2007.

[49] J. Gross, J. Kujala, M. Hamalainen, L. Timmermann, A. Schnitzler, and R. Salmelin, "Dynamic imaging of coherent sources: Studying neural interactions in the human brain," Proc. Nat. Academy Sci., vol. 98, no. 2, pp. 694-699, 2001.

[50] T. Bengtsson, P. Bickel, and B. Li, "Curse-of-dimensionality revisited: Collapse of the particle filter in very large scale systems," in Probability and Statistics: Essays in Honor of David A. Freedman. Beachwood, OH, USA: Inst. Math. Statist., 2008, pp. 316-334.

[51] S. Goncalves, J. de Munck, J. Verbunt, and F. Bijma, "In vivo measurement of the brain and skull resistivities using an EIT-based method and realistic models for the head," IEEE Trans. Biomed. Eng., vol. 50, no. 6, pp. 754767, Jun. 2003

[52] I. Santos, J. Iglesias, E. I. Olivares, and A. Young, "Differential effects of object-based attention on evoked potentials to fearful and disgusted faces," Neuropsychologia, vol. 46, no. 5, pp. 1468-1479, 2008.

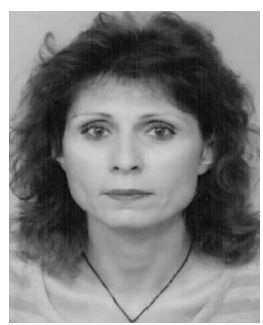

Petia Georgieva (SM'14) received the M.Sc. and $\mathrm{Ph} . \mathrm{D}$. degrees in electrical engineering from Technical University of Sofia, Sofia, Bulgaria in 1989 and 1997, respectively. Since 2003 she has been a Lecturer at the University of Aveiro, Portugal. She was visiting faculty in Computer Science Department, Carnegie Mellon University (CMU), Pittsburgh, USA, 2012, invited researcher in the Computer Science Department, University of Arkansas at Little Rock. USA, 2010, invited researcher in the School of Computing and Communications, University of Lancaster, UK, 2011. Her current research interests are in the field of machine learning with strong focus on brain study applications as Brain Machine Interface and brain neural activity recovering. Dr. Georgieva is a Senior Member of International Neural Network Society (INNS) and Elected Member of the Executive Committee of the European Neural Network Society (ENNS) for 2014-2016

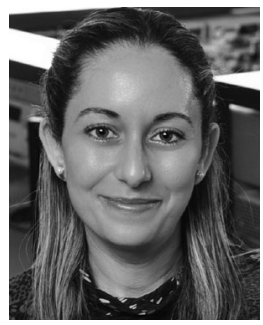

Nidhal Bouaynaya (M'08) received the B.S. degree in electrical engineering and computer science from the Ecole Nationale Supérieure de L'Electronique et de ses Applications (ENSEA), Cergy, France, in 2002, the M.S. degree in electrical and computer engineering from the Illinois Institute of Technology, Chicago, IL, USA, in 2002, the Diplôme d'Etudes Approfondies in signal and image processing from ENSEA in 2003, the M.S. degree in mathematics and the Ph.D. degree in electrical and computer engineering from the University of Illinois at Chicago,

Chicago, in 2007

From 2007-2013, she was an Assistant, then Associate Professor with the Department of Systems Engineering, University of Arkansas at Little Rock. Since 2013, she joined Rowan University, Glassboro, NJ, USA, where she is currently an Assistant Professor with the Department of Electrical and Computer Engineering. She is currently serving as an Associate Editor for EURASIP Journal on Bioinformatics and Systems Biology. Her current research interests are in biomedical signal processing, medical imaging, mathematical biology and dynamical systems

Dr. Bouaynaya won the Best Student Paper Award in Visual Communication and Image Processing 2006 and the Best Paper Award at the IEEE International Workshop on Genomic Signal Processing and Statistics 2013. 


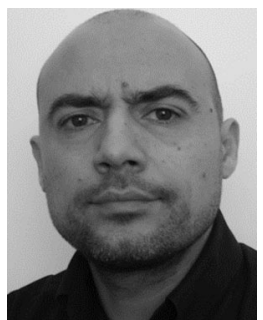

Filipe Silva received the M.Sc. degree in electronics and telecommunications engineering from the University of Aveiro, Aveiro, Portugal, in 1995, and the $\mathrm{Ph} . \mathrm{D}$. degree in electrical and computer engineering from the University of Porto, Porto, Portugal, in 2002.

Since 2003, he is an Assistant Professor with the Department of Electronics, Telecommunications and Informatics, University of Aveiro, Aveiro, and Member of the Institute of Electronics and Telematics Engineering of Aveiro, Aveiro. His current research interests include humanoid robotics, rehabilitation robotics and brain-robot interfaces.

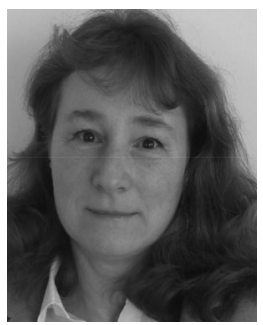

Lyudmila Mihaylova (SM'08) received M.Eng. degree in systems and control engineering, M.Sc. degree in applied mathematics and informatics, and the Ph.D. degree in systems and control engineering, from the Technical University of Sofia, Sofia, Bulgaria. She is an Associate Professor (Reader in Advanced Signal Processing and Control) with the Department of Automatic Control and Systems Engineering, University of Sheffield, Sheffield, U.K. Her interests are in the areas of autonomous systems, nonlinear filtering, tracking, statistical signal processing and sensor data fusion. She is leading a team developing novel methods and techniques in these areas, including for high dimensional problems and big data. She is a Member of the International Society of Information Fusion and an ISIF Board Member. She has been serving to the scientific community and organising a number of international conferences and symposia. She is the Associate Editor of the IEEE TRANSACTIONS ON AEROSPACE AND ELECTRONIC SYSTEMS and the Editor-in-Chief of the Open Transportation Journal. Her research is supported by EPSRC, EU and industry.

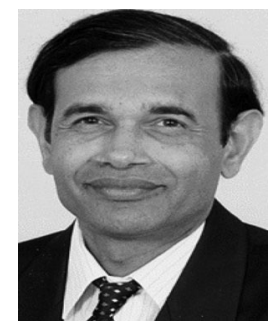

Lakhmi C. Jain received the B.E.(Hons.) and M.Eng. degrees in Electronic and Telecommunication Engineering, and the Ph.D. degree in Electronic Engineering. He is currently with the Faculty of Education, Science, Technology and Mathematics, University of Canberra, Canberra, Australia, and the University of South Australia, Adelaide, Australia. He founded the KES International for providing a professional community the opportunities for publications, knowledge exchange, cooperation and teaming. Involving around 5000 researchers drawn from universities and companies world-wide, KES facilitates international cooperation and generate synergy in teaching and research. KES regularly provides networking opportunities for professional community through one of the largest conferences of its kind in the area of KES. His interests focus on the artificial intelligence paradigms and their applications in complex systems, security, e-education, ehealthcare, unmanned air vehicles and intelligent agents.

Mr. Jain is a Fellow of the Institution of Engineers Australia. 